\title{
Lipomatosis simétrica múltiple: presentación de dos formas clínicas diferentes y revisión de la literatura
}

\author{
M. DEBÉN SÁNCHEZ, M. VELOSO ROSENDO'1, C. PÉREZ ÁLVAREZ', L. PITA \\ CAAVEIRO $^{2}$
}

Unidad Docente de Medicina de Familia. ${ }^{1}$ Servicios de Atención Primaria de Caranza y ${ }^{2}$ Cirugía General. Complejo Hospitalario Arquitecto Marcide-Profesor Novoa Santos. Ferrol, La Coruña

MULTIPLE SYMMETRICAL LIPOMATOSIS: REPORT OF TWO DIFFERENT CLINICAL TYPES AND REVIEW OF THE LITERATURE

\begin{abstract}
RESUMEN
La lipomatosis simétrica múltiple o enfermedad de Launois-Bensaude es una entidad infrecuente caracterizada por el desarrollo de depósitos grasos subcutáneos en el cuello, hombros y porción superior de extremidades. La polineuropatía periférica es un hallazgo frecuente así como su asociación a alcoholismo, hepatopatía e intolerancia a la glucosa. La patogénesis es desconocida. Describimos tres casos con dos formas clínicas distintas de presentación y revisamos la literatura médica.
\end{abstract}

PALABRAS CLAVE: Lipomatosis simétrica múltiple. Enfermedad de Launois-Bensaude. Polineuropatía.

\begin{abstract}
Multiple symmetrical lipomatosis or Launois-Bensaude disease is a rare entity characterized by the development of sucutaneous fat masses around the neck, shoulders and upper extremities. Peripheral neuropathy is a common finding as well as the association with alcholism, hepatopathy and glucose intolerance. Pathogenesis is unknown. We describe three cases with two different clinical types and review the data reported in medical literature.
\end{abstract}

KEY WORDS: Multiple symmetric lipomatosis. Launois-Bensaude disease. Polineuropathy.

Debén Sánchez M, Veloso Rosendo M, Pérez Álvarez C, Pita Caaveiro L. Lipomatosis simétrica múltiple: presentación de dos formas clínicas diferentes y revisión de la literatura. An Med Interna (Madrid) 2003; 20: 243-246.

\section{INTRODUCCIÓN}

La lipomatosis simétrica múltiple (también denominada enfermedad de Madelung, enfermedad de Launois-Bensaude o adenolipomatosis) es una entidad infrecuente caracterizada por la presencia de tejido graso no encapsulado localizado preferentemente en cuello, nuca, región supraclavicular y porción superior de extremidades. Afecta sobre todo a varones de mediana edad del área mediterránea con hábito etílico prolongado. La etiopatogenia es desconocida, aunque se ha sugerido un trastorno del metabolismo lipídico inducido por la ingesta de alcohol.

\section{CASOS APORTADOS}

En el presente trabajo describimos tres casos de esta enfermedad, con dos formas de presentación clínicamente diferentes.
Caso 1. Mujer de 46 años con prolongada historia de etilismo que consultó por aumento simétrico del perímetro de la porción superior de ambas extremidades superiores de varios meses de evolución, con aspecto de obesidad simple (Fig. 1). El cuadro se acompañaba de discreto dolorimiento e impotencia funcional en extremidades superiores.

En la analítica destacó una severa hipercolesterolemia (350 $\mathrm{mg} / \mathrm{dl})$. La radiografía de tórax resultó ser normal. La electromiografía demostró la existencia de una polineuropatía mixta de predominio axonal en ambas extremidades superiores. Se comprobó mediante biopsia la naturaleza lipomatosa de sus lesiones.

Tras abstinencia alcohólica permanecen estables las masas lipomatosas y la clínica de polineuropatía.

Caso 2. Varón de 52 años que presenta bultomas en región cervical posterior desde hace más de 5 años, los cuales han ido aumentando progresivamente de tamaño. Es fumador de más de 40 cigarrillos/día y bebedor de más de $100 \mathrm{~g}$ alcohol/día. Diagnosticado de hipertensión arterial de larga evolución, sufrió un accidente cerebrovascular lacunar izquierdo en 1999; actualmente sigue tratamiento con doxazosina y losartán.

Trabajo aceptado: 26 de marzo de 2002

Correspondencia: Martín Debén Sánchez. Unidad Docente de Medicina de Familia. Centro de Salud de Caranza. C/ Juan de Austria s/n 15406 Ferrol (La Coruña). 


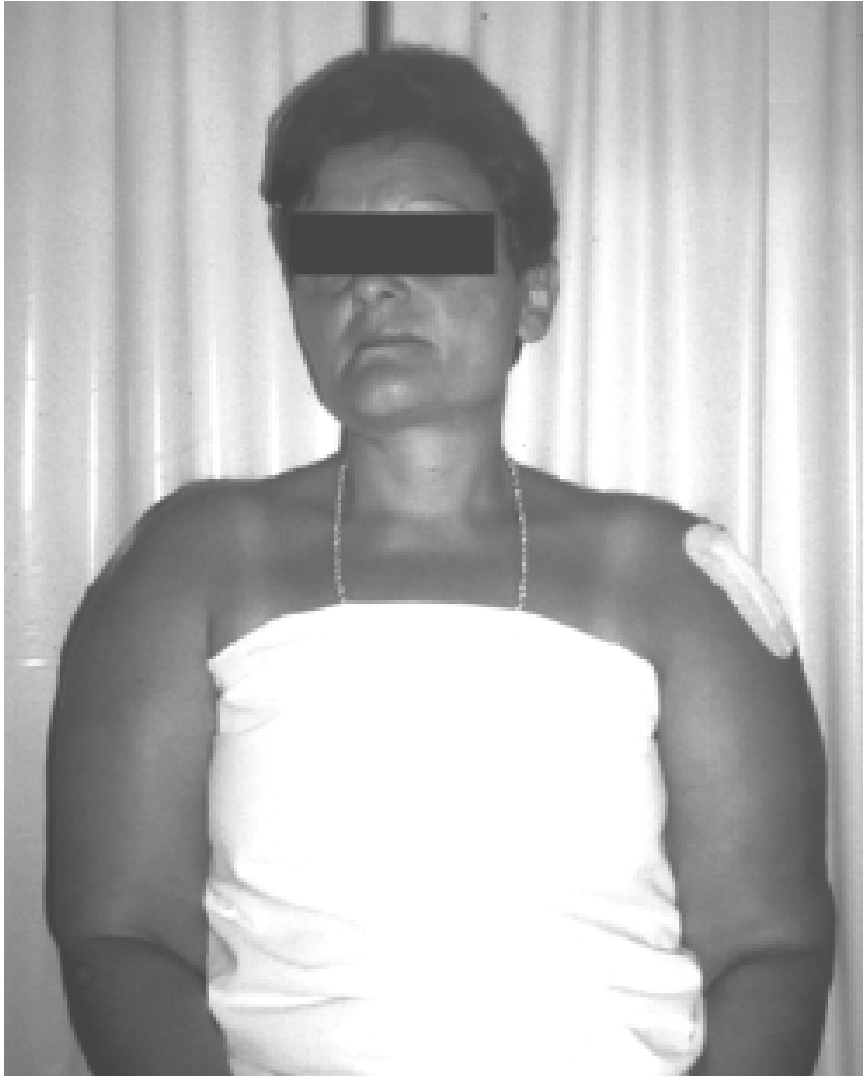

Fig. 1. Caso 1. Forma pseudoatlética: afectación de porción superior de extremidades superiores.

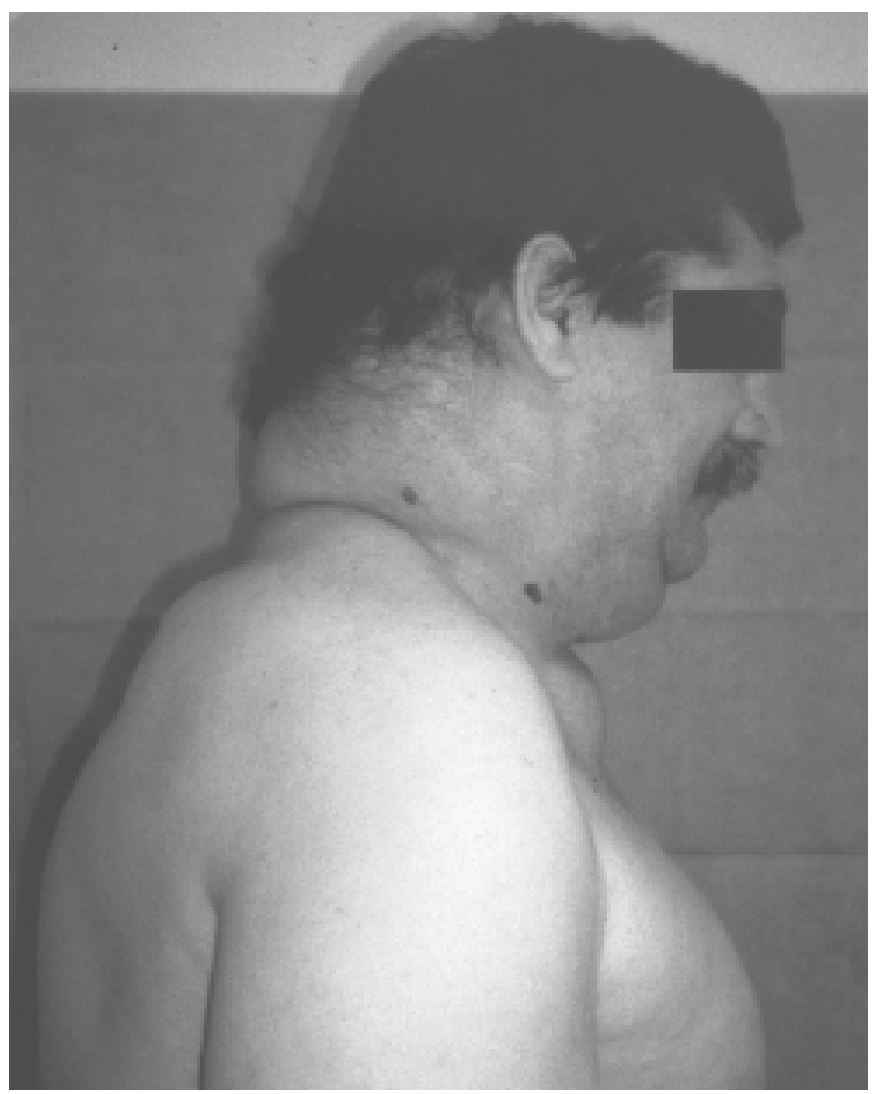

Fig. 2. Caso 2. Lipomatosis simétrica múltiple tipo M adelung.
En la exploración destacó la presencia de masas confluyentes bilaterales en la región cervical posterior, de consistencia gomosa a la palpación, no dolorosas y sin alteraciones de la piel suprayacente y otras de similares características en ambas regiones supraclaviculares (Fig. 2). No se apreciaron otras alteraciones significativas en la exploración física.

La analítica demostró VCM de 103 fl., ácido úrico de 10, 7 mgr/dl. y GGT de 83 U/L., con el resto de parámetros de función hepática, función renal y perfil lipídico normales. La radiografía de tórax y un TAC torácico fueron normales.

Caso 3. Varón de 45 años de edad con hábito etílico prolongado (más de $100 \mathrm{~g}$ alcohol/día) que presenta masas indoloras confluyentes en región cervical posterior y en ambos hombros, que habían aparecido dos años antes y aumentado de tamaño muy lentamente. No se objetivaron otras alteraciones en la exploración física, incluida una exploración neurológica sin hallazgos.

En el hemograma destacó únicamente una leve macrocitosis (VCM $99 \mathrm{fl}$ ); la bioquímica hemática demostró una GOT de 39 U/L, una GPT de $47 \mathrm{U} / \mathrm{L}$ y una GGT de $125 \mathrm{U} / \mathrm{L}$. El colesterol total fue de $242 \mathrm{mgr} / \mathrm{dl}$ (HDL 75, LDL 112) y los triglicéridos de $273 \mathrm{mg} / \mathrm{dl}$. Se solicitó una radiografía de tórax, que no demostró alteraciones significativas.

\section{DISCUSIÓN}

La primera descripción de esta entidad fue realizada por Sir Benjamin Brodie en el St. George Hospital de Londres en 1846 (1); en 1888 Otto Wilhem Madelung presenta una serie de 30 casos de lipomatosis difusa del cuello (2) y en 1898 Launois y Bensaude describen una adenolipomatosis simétrica (3). Fue denominada de varias formas: enfermedad de Madelung, lipomatosis circunscrita múltiple, lipomatosis simplex indolens, adenolipomatosis simétrica y enfermedad de Launois-Bensaude.

La enfermedad afecta predominantemente a varones alcohólicos de la cuenca mediterránea (4), pero también pueden verse afectadas mujeres, no alcohólicos y pacientes de otras razas. El comienzo suele situarse en edades medias de la vida; en series amplias varía entre los 20 y los 52 años (4). Se comunicó una incidencia de $1 / 25.000$ en la población italiana (5).

Desde el punto de vista clínico se caracteriza por la presencia de depósitos grasos no encapsulados en variadas localizaciones anatómicas (4). El curso suele ser indolente, lentamente progresivo a lo largo de meses o años, aunque se han comunicado casos de evolución mucho más rápida (6).

La etiología es desconocida. Se conoce la posibilidad de aparición familiar desde hace muchos años $(7,8)$, incluso se propuso una herencia autosómica dominante en algunos casos de esta enfermedad (9).

La biopsia de los lipomas muestra adipocitos más pequeños que los normales (10), con apariencia de tejido graso blanco, aunque se ha sugerido un origen en el tejido graso pardo y posterior migración a la localización típica de este proceso (11).

Se ha postulado la relación de la LSM con determinadas alteraciones del DNA mitocondrial $(12,13)$ y la asociación de la lipomatosis múltiple familiar con la mutación A8344 del DNA mitocondrial (14). Determinados defectos metabólicos del tejido graso (10) o la denervación de ciertas poblaciones de adipocitos (15) pueden jugar un papel importante en su patogénesis. Asimismo se han descrito disfunciones mitocon- 
driales que pueden jugar un papel patogénico importante en la presencia de neuropatía, tanto periférica como central (16), aunque no parecen presentarse de forma invariable (17).

Aunque la evolución suele ser insidiosa, inicialmente puede producirse un crecimiento rápido de las masas lipomatosas que se continúa con un período estacionario. A veces se produce un crecimiento rápido después de traumatismos o excisión de los lipomas (18).

Los lipomas se localizan predominantemente en región cervical posterior (en el tipo Madelung), hombros y porción superior de extremidades superiores e inferiores (tipo II o forma pseudoatlética), abdomen, región pélvica y caderas (tipo ginecoide) y mucho más raramente en manos $(19,20)$, pies (21) e incluso en la lengua (22-27).

Las complicaciones se derivan de la compresión de la región laringo-traqueal, del nervio recurrente $(28,29)$ y de estructuras mediastínicas, como la vena cava superior, llegando a producir síndrome de la vena cava superior $(30,31)$.

Aunque está descrita en este proceso la afectación del sistema nervioso central (32) y otras raras asociaciones (33), la manifestación neurológica más estudiada es la neuropatía periférica que cursa con afectación motora y/o sensitiva y/o autonómica. Se trata de una degeneración axonal periférica. El EMG demuestra disminución de la velocidad de conducción y la biopsia desmielinización (34). Estas alteraciones han sido atribuidas a las anomalías del metabolismo lipídico, con independencia del consumo de alcohol. Las manifestaciones propias de esta polineuropatía pueden presentarse antes que los propios lipomas (35). El inicio suele ser insidioso y su evolución progresiva y, en la mayoría de los casos, incapacitante (4). La polineuropatía periférica en el contexto de la lipomatosis simétrica múltiple está descrita en familias $(36,37)$, incluso con un patrón de herencia autosómico dominante (38).

Se encontró asociación con alteraciones del metabolismo lipídico, intolerancia a hidratos de carbono, alteraciones de la función hepática, macrocitosis con o sin anemia, que son asociaciones casuales o atribuibles al etilismo (4); está también descrita su asociación a hiperuricemia y gota (39) acidosis tubular renal (40), síndrome nefrótico (41) y púrpura trombocitopénica idiopática (42)

Está descrita la degeneración maligna histológicamente comprobada de un lipoma a un liposarcoma mixoide en un paciente que había experimentado un crecimiento asimétrico en uno de sus lipomas (43) y la asociación de este proceso con determinados tumores de cabeza y cuello (44).

Recientemente se ha descrito la asociación entre el tratamiento con inhibidores de la proteasa (indinavir) y la aparición de LSM, caracterizado por adiposidad central, dislipemia y resistencia a la insulina (45), probablemente derivado de toxicidad mitocondrial inducida por este tipo de fármacos (46, 47).

El tratamiento de la lipomatosis simétrica múltiple es a menudo insatisfactorio, puesto que el cese de consumo de alcohol no demuestra tener un efecto beneficioso definitivo y por las dificultades del tratamiento quirúrgico debido al tamaño de las masas lipomatosas y su localización.

Se ha comunicado la regresión de las masas lipomatosas con la administración de salbutamol oral (48), debido a un enlentecimiento en la acumulación de tejido graso y a un aumento en la lipolisis, tanto utilizado solo como en combinación con la cirugía.

Asimismo se ha comunicado recientemente la regresión de lipomas localizados en la pared abdominal tras la inyección intralesional de enoxaparina, probablemente mediada por la inducción de un efecto lipolítico dosis-dependiente por parte de este fármaco, aunque el mecanismo subyacente no parece totalmente claro (49).

La excisión completa de los lipomas es difícil debido a la falta de encapsulación, el incremento en la vascularización y la presencia de estroma fibroso en el tejido (50). Este tratamiento está especialmente indicado en casos de afectación funcional o posible compromiso de estructuras vitales (51) La liposucción es otra alternativa válida en determinados casos; conlleva un menor riesgo que la lipectomía, a menudo con menor número de efectos adversos, buenos resultados estéticos y la posibilidad de ser practicada de manera repetida $(52,53)$, incluso mediante apoyo ecográfico para su realización (54).

La recurrencia es frecuente tanto después del tratamiento quirúrgico convencional como de la liposucción (55) por lo que se debe considerar cuidadosamente la localización de los lipomas antes de decidirse por una técnica u otra (56).

\section{Bibliografía}

1. Brodie BC. Clinical Lectures on Surgery. Delivered at St. George's Hospital. Philadelpia, Lea \& Blanchard, 1846, pp 201-202.

2. Madelug O. Über den Fetthals(diffuses Lipom des Halses). Arch Klin Chir 1888; 37: 106-130.

3. Launois P, Bensaude R. De l'adéno-lipomatose symétrique: Bull Soc Méd Hôp 1898; 1: 298-318.

4. Enzi G. Multiple symmetric lipomatosis: an updated clinical report. Medicine 1984; 63: 56-64.

5. Enzi G, Biondetti PR, Fiore D, Mazzoleni F. Evaluation by computed tomography of the deep locations of fat masses in multiple symmetric lipomatosis. Radiology 1982; 144: 121-124.

6. Villaverde V, Garcés MC, Robayna MG, Gómez Cerezo J, Barbado FJ, Casado M. Lipomatosis simétrica múltiple: a propósito de un caso de rápida evolución. An Med Interna (Madrid) 1997; 14: 431.

7. Michon P. Adénolipomatose symétrique familiale. Presse Méd 1936; 44: 663 .

8. Kurzweg FT, Spencer L. Familial multiple lipomatosis. Am J Surg

1951; 82: 762 .

9. McKusick VA. Mendelian inheheritance in man. Baltimore: The Johns Hopkins University Press. p. 242, 1978.

10. Nielsen S, Levine J, Clay R, Jensen MD. Adipose tissue metabolism in benign symmetric lipomatosis. J Clin Endocrinol Metab 2001; 86: 2717-2720.

11. Zancanaro C, Sbarbati A, Morroni M, Carraro R, Cigolini M, Enzi G Cinti S. Multiple symmetric lipomatosis: ultraestructural investigation of the tissue and preadipocytes in primary culture. Lab Invest 1990; 63: 253-258.

12. Klopstock T, Naumann M, Schalke B, Bischof F, Seibel P, Kottlors $\mathrm{M}$ et al. Multiple symmetric lipomatosis: abnormalities in complex IV and multiple deletions in mitocondrial DNA. Neurology 1994; 44: 862-866.

13. Klopstock T, Naumann M, Seibel P, Shalke B, Reiners K, Reichman H. Mitochondrial DNA mutations in multiple symmetric lipomatosis. Mol Cell Biochem 1997; 174: 271-275. 
14. Gámez J, Playan A, Andreu AL, Bruno C, Navarro C, Cervera C et al. Familial multiple lipomatosis associated with the A8334 mutation of mitochondrial DNA. Neurology 1998; 51: 258-260.

15. Kodish ME, Alsever RN, Block MB. Benign symmetric lipomatosis: functional sympathetic denervation of adipose tissue and possible hypertrophy of brown fat. Metabolism 1974; 23: 937-945.

16. Berkovic S, Andermann F, Shoubridge E, Carpenter S, Robitaille Y, Andermann E et al. Mitochondrial dysfunction in multiple symmetrical lipomatosis. Ann Neurol 1991; 29: 566-569.

17. Mathews PM, Squier MV, Chalk C, Donaghy M. Mitochondrial abnormalities are not invariably present in neurologic syndromes associated with multiple symmetric lipomatosis. Neurology 1995; 45: 197-198.

18. Paredes BE, Braathen LR, Brand CU. Benign symmetrical lipomatosis-One cutaneous manifestation of alcoholism. Dermatology 1999; 198: 436-438.

19. Findlay GH, Duvenage M. Acquired symmetrical lipomatosis of the hands-a distal form of the Madelung-Launois- Bensaude syndrome. Clin Exp Dermatol 1989; 14: 58-59.

20. Yamamoto K, Ichimiya M, Hamamoto Y, Muto M. Benign symmetrical lipomatosis of the hands. J Dermatol 2000; 27: 748-749.

21. Requena L, Hasson A, Arias D, Martin L, Barat A. Acquired symmetric lipomatosis of the soles. A plantar form of the Madelung-Launois-Bensaude syndrome. J Am Acad Dermatol 1992; 26 (5 Pt 2): 860-862.

22. Pisanty S. Bilateral lipomas of the tongue. Oral Surg Oral Med Oral Pathol 1976; 42: 451-453.

23. Ogawa A, Nakamura H, Takahashi H. Benign symmetric lipomatosis of the tongue: report of a case. J Oral Maxillofac Surg 1988; 46: 502-504.

24. Katou F, Shirai N, Motegi K, Satoh R, Satoh S. Symmetrical lipomatosis of the tongue presenting as macroglosia. Report of two cases. J Craniomaxillofac Surg 1993; 21: 298-301.

25. Viguier J, de Muret A, Huttenberger B, Pruvost F, Vaillant L, Lorette G. Benign symmetrical lipomatosis of the tongue. Ann Dermatol Venereol 1996; 123: 814-816.

26. Ghislain PD, Garzitto A, Legout L, Alcaraz I, Creusy C, Modiano P. Symmetrical benign lipomatosis of the tongue and Launois-Bensaude lipomatosis. Ann Dermatol Venereol 1999; 126 (2): 147-149.

27. Vargas-Díez E, Dauden E, Jones-Caballero M, García-Díez A. Madelung's disease involving the tongue. J Am Acad Dermatol 2000; 42 (3): 511-513.

28. Birnholz JD, Macmillan AS Jr. Advanced laryngeal compression due to diffuse, symmetric lipomatosis (Madelung's disease). Br J Radiol 1973; 46: 245-249.

29. Borges A, Torrinha F, Lufkin RB, Abemayor E. Laryngeal involvement in multiple symmetric lipomatosis: the role of computed tomography in diagnosis. Am J Otolaryngol 1997; 18: 127-130.

30. López Aldeguer J, Portilla J, Massuti B, Caballero M. Síndrome compresivo mediastínico secundario a lipomatosis simétrica múltiple. Med Clin (Barc) 1986; 87: 779-780.

31. Fernández Sanromán J, Díaz González F, Rodríguez Campo F. Benign symmetrical lipomatosis with mediastinal involvement and growth retardation. J Oral Maxillofac Surg 1992; 50: 299-301.

32. Naumann M, Schalke B, Klopstock T, Reichman H, Lange KW, Wiesbeck $G$ et al. Neurological multisystem manifestation in multiple symmetric lipomatosis: A clinical and electrophysiological study. Muscle Nerve 1995; 18: 693-698.

33. Nounla J, Rolle U, Grafe G, Krailing K. Benign symmetric lipomatosis with myelomeningocele in an adolescent: An uncommon asscociation-case report. J Pediatr Surg 2001; 36: 13

34. Pollock M, Nicholson GI, Nukada H, Cameron S, Frankish P. Neuropathy in multiple symmetrical lipomatosis. Brain 1988; 111 (Pt 5): 1157-1171.
35. Saiz Hervas E, Martín Llorens M, López Álvarez J. Peripheral neuropathy as the first manifestation of Madelung's disease. Br J Dermatol 2000; 143: 684-686.

36. Chalk CH, Mills KR, Jacobs JM, Donaghy M. Familial multiple symmetric lipomatosis with peripheral neuropathy. Neurology 1990; 40: 1246-1250.

37. Muñoz Fernández C, Aladro Y, Conde MA, Campos Y, Arenas J. Lipomatosis simétrica múltiple con polineuropatía familiar. Rev Neurol 2001; 32: 1107-1111.

38. Stoll C, Alembik Y, Truttmann M. Multiple familial lipomatosis with polineuropathy, an inherited dominant condition. Ann Genet 1996; 39: 193-196.

39. Greene ML, Glueck CJ, Fujimoto WY. Benign symmetric lipomatosis with gout and hyperlipoproteinemia. Am J Med 1970; 48: 239246.

40. Springer HA, Whitehouse JS . Launois-Bensaude adenolipomatosis Plast Reconstr Surg 1972; 50: 291-294.

41. Matamala Gimeno C, Pérez Calvo JI, Rodríguez Benavente AM, Moros M, Pérez Villarroya JC, Bueno Gómez J. Síndrome nefrótico asociado a lipomatosis simétrica múltiple. An Med Interna (Madrid) 1995; 12: 127-129.

42. Artiles Vizcaino JA, Suarez Ortega S, Conde Martel A, Gonzalez Sanso A. Lipomatosis simétrica múltiple asociada a púrpura trombocitopénica idiopática. An Med Interna (Madrid) 1996; 13: 152.

43. Tizian C, Berger A, Vykoupil KF. Malignant degeneration in Madelung's disease (benign symmetric lipomatosis of the neck): a case report. Br J Plast Surg 1983; 36: 187-189.

44. Chan ES, Ahuja AT, King AD, Lau WY. Head and neck cancers associated with Madelung's disease. Ann Surg Oncol 1999; 6: 395397.

45. Hengel RL, Watts NB, Lennox JL. Benign symmetric lopomatosis associated with protease inhibitors. Lancet 1997; 350: 1596.

46. Brinkman K, Smeitink JA, Romijn JA, Reiss P. Mitochondrial toxicity induced by nucleoside-analogue reverse-transcriptase inhibitors is a key factor in the pathogenesis of antiretroviral therapy-related lipodystrophy. Lancet 1999; 354: 1112-1115.

47. Kakuda TN. Pharmacology of nucleoside and nucleotide reverse transcriptase inhibitor-induced mitochondrial toxicity. Clin Therapeutics 2000; 22: 685-708.

48. Leung NWY, Gaer J, Beggs D, Kark AE, Holloway B, Peters TJ. Multiple symmetrical lipomatosis (Launois-Bensaude syndrome): effect of oral Salbutamol. Clin Endocrinol (Oxf) 1987; 7: 601-606.

49. Fischer M, Wohlrab J, Taube K-M, Marsch WC. Intralesional injection of enoxaparin in benign symmetrical lipomatosis: an alternative to surgery? Br J Dermatol 2001; 144: 629-630.

50. Boozan JA, Maves MD, Schuller DE. Surgical management of massive benign symmetric lipomatosis. Laryngoscope 1992; 102: 94-99.

51. Adamo C, Vescio G, Battaglia M, Gallelli G, Musella S. Madelung's disease: case report and discussion of treatment options. Ann Plast Surg 2001; 46: 43-45.

52. Carlin MC, Ratz JL. Multiple symmetric lipomatosis: treatment with liposuction. J AM Acad Dermatol 1988; 18: 359-362.

53. Samdal F, Kleppe G, Tonvang G. Benign symmetric lipomatosis of the neck treated by liposuction. Case report. Scand J Plast Reconstr Surg Hand Surg 1991; 25: 281-284.

54. Faga A, Valdatta LA, Thione A, Buoro M. Ultrasound assisted liposuction for the palliative treatment of Madelung's disease: a case report. Aesthetic Plast Surg 2001; 25: 181-183.

55. John DG, Fung HK, van Hasselt CA, King WWK. Multiple symmetrical lipomatosis in the neck. Eur Arch Otorhinolaryngol 1992; 249 : 277-278.

56. Martínez Escribano JA, González R, Quecedo E, Febrer I. Efficacy of lipectomy and liposuction in the treatment of multiple symmetric lipomatosis. Int J Dermatol 1999; 38: 551-554. 